\title{
Thyroxinsubstitution bei subklinischer Hypothyreose
}

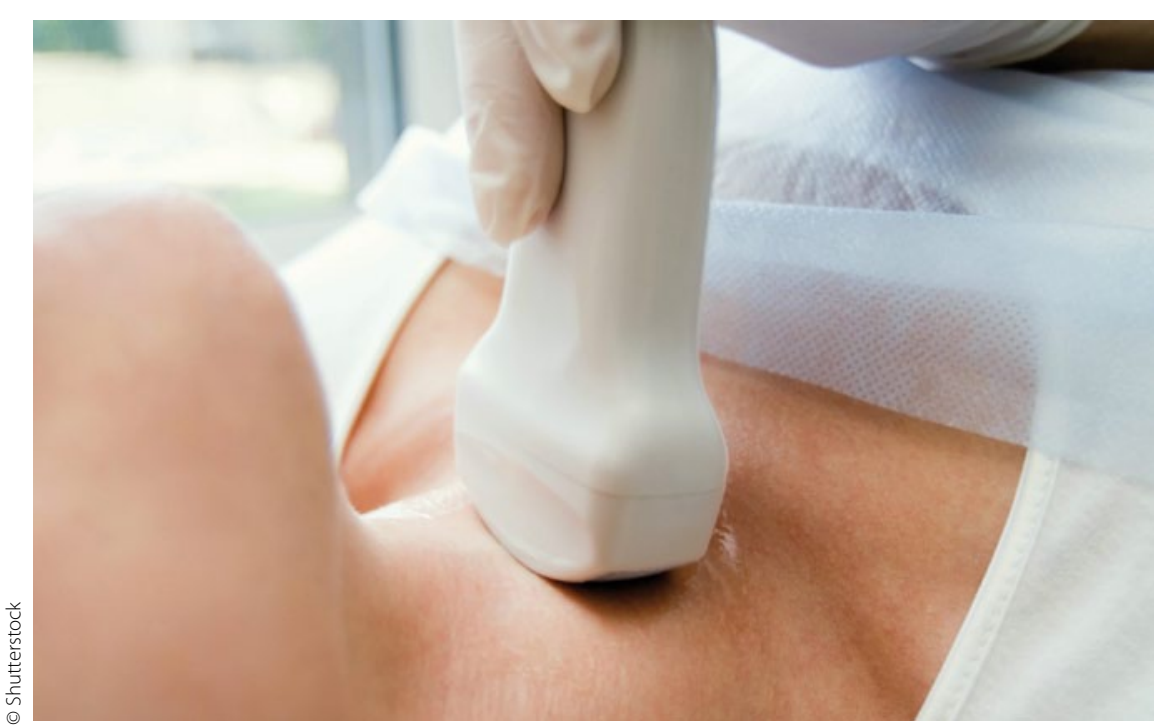

Auch wenn sie klinisch unauffällig ist, ist das kardiale Risiko bei Hypothyreose erhöht.

Wenn Patienten mit subklinischer Hypothyreose mit Levothyroxin substituiert werden, scheint dies bei Patienten unterhalb, nicht aber oberhalb des 70. Lebensjahres koronare Komplikationen zu verhindern und die Sterblichkeit zu verringern.

— Die Datenbank der Allgemeinärzte in England (GPRD) enthält die Daten von mehr als 10 Millionen Patienten, entsprechend 16\% der Allgemeinbevölkerung. Im Kalenderjahr 2001 wurden 322291 TSH-Bestimmungen in 314 Praxen gemacht. Patienten, die Schilddrüsenhormone oder antithyreoidale Medikamente erhielten, kardiovaskuläre bzw. zerebrale Erkrankungen oder andere Ausschlusskriterien hatten, wurden ausgeschlossen. Es verblieben 3093 jüngere (unter 70 Jahre) und 1642 ältere (> 70) Patienten mit einem ersten, erhöhten TSH-Wert zwischen 5,01 und 10,0 mIU/L, aber normalen T4-Werten, also einer subklinischen Hypothyreose. Im Verlauf von im Mittel 7,6 Jahre wurden $52,8 \%$ der Jüngeren und 49,9\% der Älteren mit Levothyroxin behandelt.

Bei der Analyse wurden zahlreiche Einflussfaktoren, insbesondere alle kardiovaskulären Risikofaktoren, die Höhe der TSH-Ausgangswerte und der Zeitpunkt des Beginns der Thyroxin-Therapie berücksichtigt. Bei jüngeren Pati- enten senkt die Thyroxingabe tödliche und nicht tödliche koronare Komplikationen und die Gesamtmortalität signifikant, während bei über 70-Jährigen keine signifikanten Änderungen - bei den KHK-Komplikationen sogar nachteilige Tendenzen - erkennbar sind (s. Tab. 1). Die Häufigkeit von Vorhofflimmern scheint die Levothyroxinsubstitution nicht zu begünstigen.

\section{- S. Razvi et al.}

Levothyroxine treatment of subclinical hypothyroidism, fatal and nonfatal cardiovascular events, and mortality. Arch. Intern. Med. 172 (2012) 811-817

\section{Kommentar}

Mehrere Studien haben gezeigt, dass eine subklinische Hypothyreose - vor allem bei Patienten im mittleren und jüngeren Alterhäufiger mit kardiovaskulären Erkrankungen einhergeht. Deshalb wird immer wieder eine Substitution mit Schilddrüsenhormonen gefordert. Leitlinien unterstützen diese Forderung allerdings nur bei TSH-Werten über $10 \mathrm{mIU} / \mathrm{l}$. Für erhöhte Werte bis $10 \mathrm{mIU} / \mathrm{I}$ bei asymptomatischen Patienten fehlt aber bis heute ein Wirksamkeitsnachweis durch randomisierte, kontrollierte Studien. Auch die vorliegende retrospektive Studie erfüllt nicht diese strengen methodischen Voraussetzungen, zeigt aber immerhin an einem großen Patientenkollektiv und bei langer Beobachtungsdauer einen Erfolg der Hormonsubstitution bei Patienten unterhalb des 70. Lebensjahres.

H. HolzGREVE

\section{Tabelle 1}

Patienten mit Komplikationen (\%) ohne und mit Levothyroxin-Substitution bei subklinischer Hypothyreose ( $s$ = signifikant; $n s$ = nicht signifikant)

Alter 40-70 Jahre

Alter $>70 \mathrm{Jahr}$

Mit Ohne

Levothyroxin

Mit

Ohne

\begin{tabular}{l|ll|l|ll|l|}
\multicolumn{4}{|c|}{ Levothyroxin } & \multicolumn{3}{c}{ Levothyroxin } \\
KHK-Komplikationen & $6,6 \quad \mathrm{~s}$ & $4,2 \%$ & $10,7 \%$ & $\mathrm{~ns}$ & $12,7 \%$ \\
\hline Gesamtmortalität & $6,4 \% \mathrm{~s}$ & $3,4 \%$ & $40,5 \%$ & $\mathrm{~ns}$ & $35,2 \%$ \\
\hline Vorhofflimmern & $2,3 \% \mathrm{~ns}$ & $2,0 \%$ & $7,7 \%$ & $\mathrm{~ns}$ & $8,1 \%$ \\
\hline
\end{tabular}

